\title{
CRÍTICAA LA FUNDAMENTACIÓN DEL PRINCIPIO CRÍTICO MATERIAL DE ENRIQUE DUSSEL
} José Antonio Pardo*

RESUMEN: La filosofía de la liberación de Erique Dussel se hace sospechosa, para el autor, de incurrir en falacia naturalista por la presencia de los principios materiales en su ética; por ello, se lleva a cabo una crítica a su principio ético material fundamental y, en particular, al principio ético material crítico.

PALABRAS Clave: Dussel, principio crítico-material, filosofía de la liberación, crítica, ética, falacia naturalista.
ABSTRACT: The author suspects that Enrique Dussel's philosophy of liberation is committing a naturalistic fallacy due to the presence of material principles in his ethics. In consequence, the foundation of his ethical material principle comes under scrutiny, in particular his critical material principle of ethics.

KEYWORDS: Dussel, critical material principle, Philosophy of Liberation, criticism, ethics, naturalistic fallacy.

* Departamento de Filosofía, Universidad Iberoamericana. 


\section{CRÍTICA A LA FUNDAMENTACIÓN DEL PRINCIPIO CRÍTICO MATERIAL DE ENRIQUE DUSSEL}

1. Paradójicamente, la filosofía de la liberación es prácticamente desconocida dentro del medio filosófico mexicano. Digo que es paradójico porque el más conspicuo autor dentro de dicha filosofía lleva casi cuarenta años produciendo obra filosófica en México. Me refiero a Enrique Dussel. No es la ocasión de explicar la razón de dicho desconocimiento; desde luego, es un fenómeno digno de explicación, toda vez que fuera de México la obra de Enrique Dussel no sólo es ampliamente discutida y reconocida, sino considerada acreedora de honores académicos varios. No son escasos, en universidades europeas y estadunidenses, los trabajos de doctorado dedicados al estudio de la obra de Dussel. Incluso dentro de ellos existen ya un par de monografías clásicas, a saber, la de Eduardo Mendieta en la New School for Social Research de Nueva York y la de Hans Schelkshorn en la Universidad de Viena; de la importancia del pensamiento de Dussel da cuenta también el amplio y generoso diálogo entablado durante casi diez años entre él mismo y Karl Otto Apel, documentado en un libro recientemente publicado por la editorial Trotta, así como el abrumador número de traducciones de su obra, al inglés, al francés, al portugués, al alemán, al coreano, al ruso, etc. No obstante, si bien no es ahora la ocasión de ofrecer alguna explicación, como ya dije, quizá no esté de más sugerir que tal desconocimiento no sea sino efecto de algu- 
na telaraña de prejuicios. Prejuicios tales como que Enrique Dussel no hace filosofía, sino ideología política o teología de la liberación.

Sostengo, en cambio, que Enrique Dussel es autor de una filosofía de veras, que incluso llega a abordar temas de tan profunda y acendrada estofa filosófica que aun muchas autoridades, dentro del ámbito de la filosofía política actual, ni remotamente barruntan. En seguida pretendo mostrar, a propósito de una simple tesis dentro de la filosofía de la liberación, el indiscutible talante filosófico de la obra dusseliana. Pero también expresar mi desacuerdo, de naturaleza también estrictamente filosófica, a propósito de dicha tesis. No está de más aclarar el punto. No soy dusseliano; estimo que Dussel, en relación con la tesis que ahora expongo, se equivoca. Pero una cosa es criticar una tesis filosófica, lo cual ya de suyo da testimonio del reconocimiento de la calidad intelectual del autor de la misma, y otra cosa es ese feo vicio, deplorado por Octavio Paz, del ninguneo. Este texto es una crítica a Dussel, pero una crítica que, en la medida en que es una crítica filosófica honesta, admite en el interlocutor una muy honrosa cualidad, la de ser criticable.

2. Los principios materiales de la ética son importantísimos pilares de la filosofía de la liberación; empe- ro, la presencia de los mismos hacen de ésta una filosofía sospechosa de incurrir en falacia naturalista. Enrique Dussel se hace cargo frontalmente de la posible acusación de que la filosofía de la liberación incurre en falacia naturalista en un sugerente ensayo que adoptaré como eje de la presente reflexión. Sostengo que Dussel logra superar de manera exitosa la posible acusación de incurrir en falacia naturalista sólo parcialmente. Me explico enseguida: Dussel defiende la existencia no sólo de uno, sino de dos principios materiales, a saber "el principio ético material fundamental" y "el principio ético material crítico". Lo que por mi cuenta defiendo es que Dussel sólo atina a evitar la falacia naturalista con la formulación del primero, pero no con la del segundo. Pero además, también sostengo que, para evitar dicha falacia a la hora de formular el segundo, habría que contraer un compromiso ontológico de índole idealista, idealista al modo hegeliano, compromiso que el propio Dussel en varios lugares rechaza muy ásperamente, particularmente a propósito de la obra de Porfirio Miranda. Habría que remitir a la obra de Dussel, Ética de la liberación en la Edad de la Globalización y de la Exclusión, a quien tenga curiosidad por conocer cuáles son los dos principios de los que hablo, conocimiento que, por lo demás, resul- 
NOTAS

ta indispensable para entender mi texto. Pero por cortesía expongo brevemente la formulación de ambos principios.

El principio material fundamental lo expresa Dussel así: "El que actúa éticamente debe producir, reproducir y desarrollar autorresponsablemente la vida concreta de cada sujeto humano, en una comunidad de vida". ${ }^{1}$ Por otra parte, el principio material crítico consiste en lo que sigue: "Debe ser criticado todo sistema institucional (o acto, etc.) que no permite vivir a sus víctimas, potenciales miembros negados, excluidos del sistema que tiene la pretensión de reproducir la vida". ${ }^{2}$ La justificación del primer principio, a juicio de Dussel, es fáctica, en tanto reconoce que de hecho "la producción, reproducción y desarrollo de la vida humana" se da en cada sujeto humano. ${ }^{3}$ La justificación del segundo principio depende de la del primero: si el principio material no se cumple, y el caso es que dicho principio debe ser cumplido, según el mismo primer principio, entonces debe ser criticado aquello que impide dicho cumplimiento. Dussel se demora en reforzar la tesis de que, en efecto, todo

${ }^{1}$ Enrique Dussel, Ética de la liberación en la Edad de la Globalización y de la Exclusión, 2008, Madrid, Trotta, p. 140.

${ }^{2}$ Dussel, Hacia una filosofía política crítica, 2001, Bilbao, Desclée de Brower, p. 82.

${ }^{3}$ Dussel, Ética de la liberación..., op. cit., p. 138. sujeto humano está inclinado, fundamentalmente por pulsiones de naturaleza cerebral, a conservar, producir y desarrollar su vida, cosa que hace exitosamente, y además en demostrar que es una verdad fáctica de carácter universal. Pero la transición de este hecho, universal si se quiere, al principio ético mismo, parece ser una incursión típica dentro del terreno de la falacia naturalista.

3. Es necesario distinguir para no confundir, siguiendo puntualmente la sugerencia de Dussel, la presencia de cuatro contenidos distintos, aunque yo añadiría que semejantes, según analogía de atribución, subsumidos bajo la expresión "falacia naturalista". Estos contenidos son, según las palabras del mismo Enrique Dussel: 1. El texto de Hume en que se "sugiere la diferencia entre el 'es' y el 'deber ser"'; 2. La errada identificación analítica entre atributos morales y atributos "naturales", señalada por Moore; 3. La imposibilidad de la deducción analítico-formal de juicios normativos a partir de juicios fácticos, siendo este último contenido el analogado principal en relación con los otros dos contenidos; y, 4. "La cuestión ética práctico-material que problematiza los diversos modos en que pueda efectuarse la descripción del 'pasar', por 'explicitación', desde alguna dimensión del 'ser humano' al 'deber-ser' ético". ${ }^{4}$ En realidad,

${ }^{4}$ Ibid., p. 87. 
el último no es tanto un contenido incluido bajo la expresión "falacia naturalista" como la estrategia de Dussel para resolver el problema de tal falacia.

A propósito del primer contenido, Dussel sostiene, siguiendo muy de cerca a MacIntyre, que en realidad Hume no habla para nada de falacia naturalista, sino que, por el contrario, en realidad diseña una ética fundada en la facticidad de los sentimientos morales. Detengámonos en el texto humeano.

Me sorprende encontrar que en vez de la cúpula usual de las proposiciones, 'es' y 'no es', no doy con ninguna proposición [en el terreno de la ética] que no esté conectada con un 'debería' o con un 'no debería'. Este cambio es imperceptible, pero es, sin embargo, la última consecuencia. Puesto que este 'debería' o 'no debería' expresa una nueva relación o afirmación, es necesario que fuese observado y explicado, y al mismo tiempo que fuese ofrecida una razón para aquello que en general parece inconcebible (seems inconceivable), cómo esta nueva relación puede ser una deducción de otras que son completamente diferentes de ésta. ${ }^{5}$

MacIntyre afirma que en este lugar Hume en realidad no desautoriza la

${ }^{5}$ David Hume, Treatise on Human Nature, III, i, i, 1969, London, Harmondsworth, p. 521. transición del 'es' al 'debería', sino que simplemente afirma que aunque "parece inconcebible" tal transición, aunque sea una "transición difícil" que nadie ha atinado a concebir, puede, sin embargo, llevarse a cabo, y que en realidad el propósito de la redacción del resto del libro III del Tratado consiste en "mostrarnos cómo es posible hacerla (show us how it can be made)". ${ }^{6}$ Hume, pues, dice MacIntyre, "no está intentando decir que la moralidad carece de fundamento, él está más bien intentando puntualizar cuál es la naturaleza de ese fundamento". ${ }^{7}$ De ello Dussel concluye que "No sólo Hume no habla de la 'falacia naturalista' (en sentido estricto, como en 3 , más adelante), sino que por el contrario, nos está diciendo que el 'deberser (ought)' ético se infiere o fundamenta en el 'es (is)', entendido éste en el nivel de los sentimientos, el placer, la felicidad, las pasiones". ${ }^{8}$

Por mi parte, opino que esta interpretación del texto humeano me resulta convincente. Aun así, es verdad, tal como Dussel reconoce, que la fundamentación de la moral llevada a cabo por Hume no es una fundamentación racional. No me extende-

${ }^{6}$ A.C. MacIntyre, "Hume on 'is' and 'ought"”, en Philosophical Review, núm. 68, 1959, Duke University Press, p. 463.

${ }^{7}$ Ibid., p. 466.

${ }^{8}$ Dussel, Hacia una filosofía politica crítica, p. 89. 
NOTAS

ré sobre el asunto pero, por apelar a una fundamentación no racional, Dussel estaría incurriendo en una falacia de re-definición a propósito del uso de la palabra "fundamentación". Es decir, que la fundamentación no sea racional no significa que Hume no ofrezca razones que justifiquen su postura, sino que "la distinción entre vicio y virtud no está fundada sólo en relaciones entre objetos, ni puede ser percibida por la razón". ${ }^{9}$ Pero si la distinctión entre "virtud" y "vicio" no está fundada sobre relaciones de hechos, aunque pueda ser explicada racionalmente, explicación que desde luego Hume intenta ofrecer, empero no estará fundamentada.

Por otra parte, sospecho que si Dussel y MacIntyre tienen razón, lo consecuencia es punto menos que paradójica, a saber, que Hume, en efecto, no denuncia ninguna falacia naturalista, pero sí incurre en ella. No es fácil afirmar tal cosa, que Hume incurre en falacia naturalista, pero mejor demorarse en esto en otra ocasión.

4. A propósito del segundo contenido, Dussel censura un posible reduccionismo falaz en Moore. Se trata de un reduccionismo que por mi cuenta sí encuentro, aunque no me satisface la descripción que del mismo hace Dussel. Escribe, en efecto,

${ }^{9}$ David Hume, Ibid. que Moore no distingue entre la "referencia" de los enunciados descriptivos y la de los enunciados normativos. Escribe éste, en efecto, que Moore comete una "falacia reduccionista" porque "no distingue entre la 'referencia' de los enunciados descriptivos (dirigida teóricamente a objetos del mundo) y la de los enunciados normativos (dirigida prácticamente a exigencias del sujeto humano situado en una estructura intersubjetiva, cultural, histórica)". ${ }^{10}$ Para explicar la razón de mi insatisfacción con esta descripción permítaseme tomar el salto desde más atrás. Moore sostiene que el concepto "bien" es inanalizable mediante el uso de atributos naturales, por ejemplo diciendo que "bueno" significa "placentero", porque de no ser así, entonces una proposición tal como "el placer es bueno", en virtud del principio de indiscernibilidad de los idénticos, sería equivalente a "el placer es el placer", lo cual no es el caso. Es decir, dado que los enunciados "el placer es el placer" y "el placer es bueno" no tienen el mismo valor semántico, por lo tanto no pueden compartir la misma referencia. En realidad lo que Moore llama "falacia naturalista" consiste en no distinguir las referencias propias de los enunciados descriptivos de la de los normativos, de modo que es un error acusar a Moore de no

\footnotetext{
${ }^{10}$ Ibid., p. 91.
} 
hacer esta distinción. Por lo demás, parte de la argumentación del mismo Dussel descansa sobre la tesis de que los enunciados normativos y los enunciados fácticos en realidad se refieren a lo mismo, a saber a "la vida humana", en cuanto ésta es, según escribe Dussel "criterio de verdad práctica y teórica”. Esto último se entenderá mejor después de lo que sigue.

La dificultad planteada por Moore a propósito del análisis del concepto "Bien" es exactamente la misma que se presenta para el análisis de cualquier otro concepto. A esta dificultad, Langford le ha llamado "paradoja del análisis". ${ }^{11}$ Tal paradoja consiste en que, para todo análisis, si lo que expresa el definiens no es lo mismo que expresa el definiendum entonces el análisis es falso; pero si lo que expresa uno y lo que expresa el otro son lo mismo, entonces el resultado es un enunciado trivial. Alonzo Church, ${ }^{12}$ haciendo uso de la distinción de Frege entre sentido y referencia, ${ }^{13}$ resuelve la paradoja defendiendo que, para todo análisis

${ }^{11}$ C.H. Langford, "Notion of Analysis in Moore's Philosophy", en Paul Arthur Schilpp (edit.), The Philosophy of G.E. Moore, 1992, Illinois, La Salle, p. 323.

${ }^{12}$ Alonzo Church, "A note on "The Paradox of Analysis by Morton White", en The Journal of Symbolic Logic, 1946, vol. 11, núm. 4, p. 133.

${ }^{13}$ Gottlob Frege, "Sobre sentido y referencia", en Ensayos de semántica y filosofía de la lógica, 1998, Madrid, Tecnos, pp. 84 ss. correcto, el definiens y el definiendum se refieren a lo mismo, a saber, lo que Frege llama Bedeutung o significado, ${ }^{14}$ pero que lo hacen de manera distinta, es decir, asociando los signos que integran el definiens y el definiendum a sendos y distintos "sentidos" (Sinnen). Quiero decir, pues, que lo que Moore confunde no es la referencia de los enunciados teóricos y la de los normativos, sino el sentido. Que el descuido de Dussel es sólo léxico, y que por lo tanto no afecta seriamente a la argumentación, pues no es en realidad una inconsistencia, se echa de ver en que lo que constituye el valor de verdad de una proposición tal como “"Bueno' significa ' $x$ '”, donde ' $x$ ' está en lugar de un juicio teórico, es que 'Bueno' $y$ ' $x$ ' significan lo mismo (el Bedeutung, según Frege y Church, es el valor de verdad de la proposición en la que se expresa el análisis); a saber, de acuerdo con Dussel, la vida humana, pero de distinto modo o por medio de sendos sentidos.

Si no se admite la distinción entre sentido y significado para enunciados analíticos no triviales, y en general, si

${ }^{14}$ Peter Geach sostiene que Russell comete un error cuando piensa que para todo caso es verdad que "bedeuten" es equivalente a "denote", pues "lo que el término general 'hombre' denota, podría ordinariamente ser tomado como los hombres individules, mientras que para Frege [en el caso del análisis] el Bedeutung de 'hombre' no es muchos hombres, sino un concepto", Peter Geach, Logic Matters, p. 28. 
NOTAS

no se admite la distinción entre sentido y referencia, entonces se presenta la imposibilidad señalada por el último contenido subsumido bajo la expresión "falacia naturalista". Es sobre todo a raíz de la adopción de una semántica exclusivamente extensional, desde la semántica verificacionista del círculo de Viena hasta Quine y sus seguidores, que se ha tornado muy fácil impugnar, mediante el recurso de apelar a la falacia naturalista, la defensa de cualquier principio material para la ética.

5. La estrategia argumentativa de Dussel consiste en mostrar que la referencia a la vida humana admite tanto descripciones asociadas a "sentidos teóricos" como a "sentidos prácticos", lo cual quedaría probado si se encontrara algún tipo de enunciado que, por referirse a la vida humana, sea "al mismo tiempo" fáctico y normativo. "Deberían referirse, en el nivel material -escribe Dussel congruentemente con la enmendadura que he hecho-, a la 'vida humana' en cuanto humana". ${ }^{15}$ Dicho enunciado sería, por ejemplo: "Juan es un ser humano y, por lo tanto, cerebralmente dotado de conciencia, autoconciencia y responsabilidad sobre su vida".

En efecto, es perfectamente descriptible, de manera correcta y en términos tanto fácticos como mora-

${ }^{15}$ Dussel, op. cit., p. 94. les, "el modo de realidad" de Juan por medio de los atributos "tener cerebralmente: conciencia, autoconciencia y responsabilidad sobre la propia vida". De este modo, distintas expresiones denotativas tales como "el organismo que tiene cerebro humano" o "el organismo que tiene conciencia, autoconciencia y responsabilidad sobre la propia vida", distintas en virtud de los sendos sentidos asociados a ellas, y teniendo éste como contenido atributos morales "ser responsable" y aquél, en cambio, atributos físicos como "tener cerebro humano", admitirían ser intercambiadas sin alterar el valor de verdad (salva veritate) de las proposiciones de las cuales formen parte, en tanto se refieren ambas a la misma realidad viviente. El pasaje, pues, de lo "teórico" a lo "práctico" estaría fundado y justificado en virtud de la referencia común, en este caso, de lo teórico y de lo práctico.

La justificación de que en un caso como éste "lo teórico" y "lo práctico" se refieren a lo mismo, a la vida humana, es llevada a cabo por medio de una deducción trascendental (que Dussel llama "dialéctica"), ${ }^{16}$ cuya

${ }^{16}$ Dussel llama dialéctica, en referencia a Hegel, a la inferencia que va desde "el aparecer" hacia "el fundamento" de dicho aparecer, inferencia según la cual el aparecer sería la explicitación del implícito fundamento y, por tanto, el fundamento la explicación de su aparecer. Es un error. Me parece que no es esto, estrictamente, en lo que consiste la dialéctica hegeliana, sino en lo que con- 
conclusión es que toda acción humana, desde la elaboración de una herramienta hasta la fundación de una institución o la simple emisión de un enunciado con pretensión de objetividad, tiene como condición de posibilidad el haberse hecho cargo de la propia vida y haber decido no cometer suicidio. A propósito de esto, Dussel recurre a la autoridad de Wittgenstein, quien afirma: "Si el suicidio estuviera permitido, todo entonces está permitido [...] aunque acaso el suicidio tampoco sea, por sí mismo, ni bueno ni malo". ${ }^{17}$ Este hacerse cargo de la propia realidad, por más que Zubiri afirme que es "un hecho", se trata más bien de una hipótesis explicativa (el fundamento del fenómeno, de acuerdo con la terminología dusseliana) susceptible de demostración trascendental.

siste, entre otras cosas, la deducción de las categorías del entendimiento (deducción trascendental) practicada por Kant. Lo estrictamente dialéctico, en Hegel, es la refutación, entre otras cosas, de dicha operación llevada a cabo por Kant. El momento conclusivo de la argumentación hegeliana, posterior al momento dialéctico y que Hegel llama "especulativo", es el reconocimiento no ya de las condiciones de posibilidad del "aparecer" (lo implícito del aparecer), sino de las condiciones de posibilidad de la refutación de la deducción hecha por Kant (lo implícito en la refutación de la deducción trascendental). Acerca de esta interpretación que hago de Hegel he escrito un artículo que pronto será publicado y cuyo título es “¿Cuál debe ser el comienzo de la ciencia?".

${ }^{17}$ Texto del 10 de enero de 1917, citado por Dussel, Filosofia de la liberación ..., op. cit., p. 142.
Dussel demuestra, pues, que todo orden vigente, en la medida en que es fruto de la acción o de la praxis humana, es ya la respuesta a un imperativo práctico. Es decir, se debe de suponer la existencia de un imperativo práctico-material expresable como: ¡No te mates!, pues de no hacerse el mero modo de "aparecer" del "mundo humano" resultaría ininteligible o infundado. Este mundo, pues, en su simple y nudo acaecer implica ya, como condición implícita, la existencia, ciertamente a nivel cerebral, de lo normativo.

6. Me parece, pues, que con esto Dussel logra evitar satisfactoriamente la falacia naturalista. Empero, el principio material crítico ofrece otras dificultades, también asociadas con el nombre "falacia naturalista", que con sólo lo expuesto hasta aquí no quedan resueltas.

El principio crítico material parte, escribe Dussel, de la constatación empírica:

buena parte de la humanidad, (los miserables del sur, las naciones endeudadas, los pobres en todo sistema, las clases oprimidas, los campesinos, los inmigrantes, los marginales, los desempleados, las mujeres, los niños de la calles, los ancianos en asilos, las culturas originarias oprimidas por la Modernidad, las razas no-blancas... y toda la humanidad 
NOTAS

en peligro de extinción ecológica) no puede vivir..$^{18}$

Si hemos admitido el principio ético material, de él se sigue que el sistema que produce todas estas víctimas no puede ser verdadero $\mathrm{y}$, por ende, es digno de ser criticado y derruido. A mí, en cambio, me parece que esto no se sigue de aquello, pues la palabra "verdadero" usada en la conclusión no tiene el mismo "significado" que la palabra "verdadero" utilizada en las premisas. La prueba de que sí se da esta anfibología es que si no fuera el caso, entonces la relación entre la conclusión y las premisas sería de inconsistencia, es decir, sería necesariamente falsa.

Dussel afirma que todo orden vigente, en cuanto que existe, tiene como contenido, a la vez fáctico y normativo, "la vida humana", de modo que cualquier sistema humano, en la medida de que existe, satisface la "descripción que incluye términos normativos": "todo acto, norma o institución, etc., tiene como contenido fáctico el deber producir, reproducir y desarrollar la vida humana", y por ende, "todo acto, norma o institución, es, en sentido analógico atributivo, verdadera. Es decir: para toda $\mathrm{x}$, si $\mathrm{x}$ es un acto, o $\mathrm{x}$ es una norma, o $\mathrm{x}$ es una institución, entonces $\mathrm{x}$ es verdadera

\footnotetext{
${ }^{18}$ Ibid., p. 371.
}

(según analogía de atribución). Pero, la postura de Dussel incluye también el supuesto de que hay por lo menos una $x$ tal que no es verdadera. Es posible alegar, para evitar la inconsistencia-como de hecho hace Dussel-, que en realidad el contenido del acto, norma, institución, etc., no es tanto la producción, reproducción y desarrollo efectivo de la vida humana, como la "pretensión" de lo mismo. Es decir, no es el caso, por lo tanto, que el contenido de todo acto, norma e institución sea la "bondad" o "verdad", sino la "pretensión de bondad" o "pretensión de verdad". Es, en realidad, que por hablar de "pretensión" que se abre espacio a la falibilidad y por lo tanto a la ocasión para la crítica.

No obstante, si la pretensión de verdad se mantiene, aun cuando no se cumpla la misma, entonces es imposible establecer una relación de bicondicionalidad entre pretensión y cumplimiento de la pretensión, de suerte que sí se estaría incurriendo en falacia de anfibología: de la falsedad de A no se sigue la negación de la pretensión de A, ni, en el mismo sentido, de la negación de A se sigue la falsedad de la pretensión de A. Para justificar que el incumplimiento de una pretensión la anula como realidad, es decir, para justificar que el incumplimiento de una pretensión sea interpretable como "negatividad", es necesario 
postular un tipo de entidad de naturaleza no empírica, sino ideal, a saber el deber mismo, cuya materia es la subjetividad en sentido racionalista, lo que Hegel y Porfirio Miranda llaman "el Espíritu". Es verdad que resulta empíricamente constatable "la pretensión de verdad", aunque no sea porque dicha pretensión se postule como factor explicativo de cierta conducta empíricamente constatable, de modo que ciertas conductas de este tipo sólo resulten explicables bajo el postulado de cierta compulsión vital, voluntad de vida o voluntad tendente, tal como la llama Xavier Zubiri. Pero que la violencia contra dicha compulsión sea auténtica violencia moral (negación), y no la violencia que se ejerce, por ejemplo, contra el cuerpo cuando se le ejercita para una competencia atlética (negación de la negación), implica que el cumplimiento de la tal compulsión sea obligatorio de manera categórica y no empírica. La falta de cumplimiento de una tendencia o de una pretensión no es ningún momento negativo para dicha tendencia o para dicha pretensión en cuanto realidad, pues una tendencia o una pretensión insatisfechas, en términos fácticos, son tan realmente tendencias o pretensiones como lo son si están, en cambio, satisfechas. Dussel le reprocha a Porfirio Miranda haber confundido "la vida", con "la idea de vida". El problema es que, sin esta "confusión", entonces "el principio vida" se torna incapaz de generar, de manera consistente, ningún principio material crítico. 
CITAM Derechos Reservados.

La reproducción total o parcial de este artículo se podrá hacer si el ITAM otorga la autorización previamente por escrito. 